\title{
Translation and Validation of the Indonesian MD Anderson Dysphagia Inventory (MDADI) in Head and Neck Cancer Patients with Swallowing Disorders
}

\author{
Rizka Fakhriani ${ }^{1,2}$ \\ Agus Surono ${ }^{2}$ \\ ${ }^{1}$ Departement of Otorhinolaryngology, Head and Neck Surgery, \\ Faculty of Medicine and Health Science, University of \\ Muhammadiyah Yogyakarta, Yogyakarta, Indonesia \\ 2 Departement of Otorhinolaryngology Head and Neck Surgery, \\ Faculty of Medicine, Public Health and Nursing, University of Gadjah \\ Mada, Yogyakarta, Indonesia
}

Bambang Udji Djoko Rianto 2 (1)

Int Arch Otorhinolaryngol 2022;26(3):e321-e326.

\author{
Address for correspondence Rizka Fakhriani, Departement of \\ Otorhinolaryngology Head and Neck Surgery, Faculty of Medicine and \\ Health Science, University of Muhammadiyah Yogyakarta, Jl. \\ Brawijaya, Kasihan, Bantul, Yogyakarta 55183, Indonesia \\ (e-mail: rizkafakhriani@umy.ac.id).
}

\begin{abstract}
Keywords

- dysphagia

- quality of life

- head and neck cancer

- survey and questionnaire

Introduction Dysphagia is common in head and neck cancer patients; it is associated with significant morbidity, including quality of life. Several instruments can be used to assess the quality of life of dysphagia patients, including the M.D Anderson dysphagia inventory (MDADI) questionnaire, which is sufficiently valid and reliable to improve the quality of life of patients with neurological disorders and head and neck cancer.

Objective The purpose of the present study is to perform adaptation, cultural translation, and validation of the MDADI questionnaire for the Indonesian language.

Methods This cross-sectional study assessed the validity and reliability of the MDADI Indonesian adaptation instrument in head and neck cancer patients with swallowing disorders in the Otorhinolaryngology clinic of the Dr. Sardjito hospital, Yogyakarta, from May to August 2019.

Results There were 40 study subjects, including 31 men and 9 women. The MDADI instrument adapted to Indonesian is valid and reliable as an instrument for assessing the quality of life of patients with head and neck cancer with swallowing disorders, with r-values ranging from 0.314 to 0.939 . Internal consistency shows that Cronbach's $\alpha$ is 0.915 , and test-retest reliability (intra-class correlation) ranges from 0.919 to 0.985 . Conclusion The translation and validation of the Indonesian MDADI instrument were performed as an instrument for assessing the quality of life of head and neck cancer patients with swallowing disorders.
\end{abstract}

\section{Introduction}

Dysphagia, or swallowing disorder, is a common problem in almost all populations, especially in the elderly. ${ }^{1}$ Within 3 years after head and neck cancer treatments, 40\% of patients experience dysphagia. ${ }^{2}$ This condition is related to significant morbidity, including health-related quality of life (HRQoL), anxiety, and depression. ${ }^{3}$ Some of the medical complications of dysphagia are oesophageal stricture, pneumonia, and malnutrition. ${ }^{4}$ received

August 31, 2020 accepted after revision

April 22, 2021

published online

October 26, 2021
DOI https://doi.org/ 10.1055/s-0041-1735566. ISSN 1809-9777.

\footnotetext{
(C) 2021. Fundação Otorrinolaringologia. All rights reserved. This is an open access article published by Thieme under the terms of the Creative Commons Attribution-NonDerivative-NonCommercial-License, permitting copying and reproduction so long as the original work is given appropriate credit. Contents may not be used for commercial purposes, or adapted, remixed, transformed or built upon. (https://creativecommons.org/ licenses/by-nc-nd/4.0/) Thieme Revinter Publicações Ltda., Rua do Matoso 170, Rio de Janeiro, RJ, CEP 20270-135, Brazil
} 
Within the last decade, questionnaires to assess dysphagia's impact on the quality of life and to evaluate the effectiveness of treatments have been developed. These questionnaires include Swallowing Quality of Life (SWALQOL), MD Anderson Dysphagia Inventory (MDADI), and Deglutition Handicap Index, and Eating Assessment Tool (EAT-10). ${ }^{5-7}$ Chen et al. developed the MDADI questionnaire, which is reasonably valid and reliable to evaluate the impact of dysphagia on the quality of life of patients who undergo head and neck cancer treatments. ${ }^{6}$

The primary consideration to translate and validate the MDADI is the fact that it has no Indonesian translation yet. Although there are fewer questions in the MDADI than in the SWAL-QOL (44) questionnaire, the former is more convenient for patients and clinicians, and it still covers all relevant concepts. Moreover, the MDADI also shows consistency and high reliability in the head and neck cancer patient population with a Cronbach $\alpha$ score of 0.85 to 0.93 and test-retest score of 0.69 to $0.88 .{ }^{6}$ The present study translates, adapts, and validates the Indonesian version of the MDADI for head and neck cancer patients with dysphagia.

\section{Methods}

\section{Study Design}

The current cross-sectional study was conducted in the otorhinolaryngology clinic of Dr. Sardjito Hospital in Yogyakarta from May to August 2019. The Indonesian version of the MDADI questionnaire was validated, and the patients' characteristics and clinical features were detailed as shown in - Supplementary Appendix A (online only). The inclusion criteria for the study were patients who were diagnosed with head and neck cancer and experience dysphagia and whose medical records are complete, documented in detail and accurately, aged over 18 years old, and consented to participate in the study. The preliminary diagnosis of dysphagia was performed by a subjective diagnosis and emphasized by several questions, such as: In the last few months, have you had problems eating, drinking, or swallowing?; Have you coughed or choked after eating?; Do you use naso gastric tube or percutaneous endoscopic gastrostomy? The exclusion criteria for the study were patients with severe illness who cannot participate in regular activities such as unable to mobilize, in an unfavorable general condition, or do not speak Indonesian.

\section{MDADI Questionnaire}

The study began with adaptation to Bahasa Indonesia from the original MDADI questionnaire, in English. Cross-cultural adaptation was adjusted using the World Health Organization (WHO) methods until we obtained the Indonesian adaptation of the MDADI questionnaire (-Supplementary Appendix A [online only]). The WHO cross-cultural adaptation method consists of five steps, namely forward translation, expert committee meeting, back translation, a trial questionnaire with monolingual people, and thorough documentation of those processes. ${ }^{8}$ Patients who met the inclusion criteria and consented to participate in the study were assessed for their quality of life, using the MDADI questionnaire that was cross-culturally adapted to Bahasa Indonesia using the WHO criteria at the $1^{\text {st }}$ meeting and 2 weeks after the $1^{\text {st }}$ form filling.

\section{Data Analysis}

In the present study, data were processed using the following methods: 1) Descriptive presentation by calculating the frequency distribution, proportions, and the average of each variable; 2) Adaptation of cross-cultural (transcultural) questionnaires was performed by the WHO method. Research of the data that had been collected was processed using a computer program. Data analysis was performed using tests of internal validity and reliability of the adaptation of the MDADI questionnaire for the Indonesian language. The validity test was done by the Pearson correlation test, measuring the correlation between the question items and the overall question score. The significance value of $p<0.05$ indicates that the items tested were valid. Reliability tests with internal consistency were used to find the Cronbach a values. It is considered reliable if it has an $\alpha$ value of at least 0.7 .

\section{Results}

\section{Sample Characteristic}

The present study includes 40 participants with a higher number of male subjects (-Table 1); 31 male subjects (77.5\%), and 9 female subjects (22.5\%). The subjects' average age was 52.7 years old (range: $19-79$ years old). Subjects in the age group $<45$ years old were most prevalent, namely 16 (40\%). The number of subjects in the tube feeding group was higher, with 38 people (95\%), compared with 2 people (5\%) in the no tube feeding group. The most prevalent head and neck cancer group found was nasopharyngeal cancer, which included 19 people (47.5\%). The advanced stage (T3-T4) cancer group was also more prevalent, with 36 people $(90 \%)$ compared with 4 people (10\%) in the early stage (T1-T2) group. The most prevalent therapy group found was chemotherapy and radiotherapy group, which included 27 people (67.5\%).

\section{Indonesian MDADI}

The current study assessed patients on 2 occasions, at the $1^{\text {st }}$ meeting and 2 weeks after completing the questionnaire. In the initial assessment, we obtained a mean total score for the Indonesian MDADI of $32.21 \pm 6.73$, with a minimum score of 22.11 and a maximum score of 41.05 . In the second assessment, we obtained a mean total score for the MDADI of $31.45 \pm 6.42$, a minimum score of 21.05 , and a maximum score of 41.05 .

\section{Validity and Reliability of the Indonesian MDADI}

The present study performed the validity test on 40 subjects; hence, the r-value of the table was 0.312 . Based on the $\mathrm{r}$ value, we found that all of the questions in the MDADI questionnaire have greater $r$-values than the those in the Table $(0.312$ for $\mathrm{df}=38)$, indicating that the Indonesian MDADI questionnaire is valid for use. 
Translation and Validation of the Indonesian MD Anderson Dysphagia Inventory (MDADI) Fakhriani et al. 323

Table 1 Demographic and clinical features of patients

\begin{tabular}{|c|c|c|}
\hline Characteristic & Value $(n)$ & Percentage (\%) \\
\hline \multicolumn{3}{|l|}{ Gender } \\
\hline Male & 31 & 77.5 \\
\hline Female & 9 & 22.5 \\
\hline \multicolumn{3}{|l|}{ Age (years) } \\
\hline$<45$ & 16 & 40 \\
\hline $45-54$ & 7 & 17.5 \\
\hline $55-64$ & 8 & 20 \\
\hline $65-74$ & 7 & 17.5 \\
\hline$>74$ & 2 & 5 \\
\hline Mean & 52,675 & \\
\hline \multicolumn{3}{|l|}{ Feeding } \\
\hline Tube feeding & 38 & 95 \\
\hline No tube feeding & 2 & 5 \\
\hline \multicolumn{3}{|l|}{ Tumor location } \\
\hline Oropharynx & 6 & 15 \\
\hline Larynx & 9 & 22.5 \\
\hline Nasopharynx & 19 & 47.5 \\
\hline Sinonasal & 4 & 10 \\
\hline Parotid & 1 & 2.5 \\
\hline Thyroid & 1 & 2.5 \\
\hline \multicolumn{3}{|l|}{ T (TNM) } \\
\hline $\mathrm{T} 1-\mathrm{T} 2$ & 4 & 10 \\
\hline T3-T4 & 36 & 90 \\
\hline \multicolumn{3}{|l|}{ Treatment } \\
\hline Radiotherapy (RT) & 3 & 5 \\
\hline Chemoradiotherapy & 27 & 67.5 \\
\hline Surgery + radiotherapy & 11 & 27.5 \\
\hline
\end{tabular}

The reliability tests used in the current study are the internal consistency and test-retest reliability. We found that the Cronbach $\alpha$ score of the Indonesian MDADI questionnaire was 0.915 , indicating that the instrument is reasonably reliable (Cronbach $\alpha$ score $>0.7$ ). The test-retest reliability was calculated by giving an instrument to the same population on two different occasions. In the present study, we evaluated the instrument with an interval of 2 weeks.

In this study, we also performed an intraclass correlation coefficient (ICC) evaluation based on a 2-way mixed model, mean rating $(\mathrm{k}=2)$, and absolute consistency. The ICC result of the MDADI questionnaire is presented in - Table 2.

The present study was conducted using the Pearson coefficient correlation test. The result showed that the rvalue of each item, domains, and the total score was $\geq 0.7$ $(p<0.05)$, indicating that the Indonesian MDADI is a reliable instrument.

Plot and Bland-Altman analyses (mean difference or limits of agreement) were performed to compare two values for the same variable; accordingly, the method of analysis was
Table 2 Intraclass correlation coefficient (ICC) for individual domain and composite score of MDADI instrument

\begin{tabular}{|l|l|l|}
\hline Domain & ICC, mean $(95 \% \mathrm{CI})$ & Interpretation \\
\hline Global & $0.919(0.848-0.957)$ & Good-excellent \\
\hline Emotional & $0.973(0.945-0.986)$ & Excellent \\
\hline Physical & $0.978(0.958-0.989)$ & Excellent \\
\hline Functional & $0.945(0.895-0.971)$ & Good-excellent \\
\hline Composite score & $0.985(0.962-0.993)$ & Excellent \\
\hline
\end{tabular}

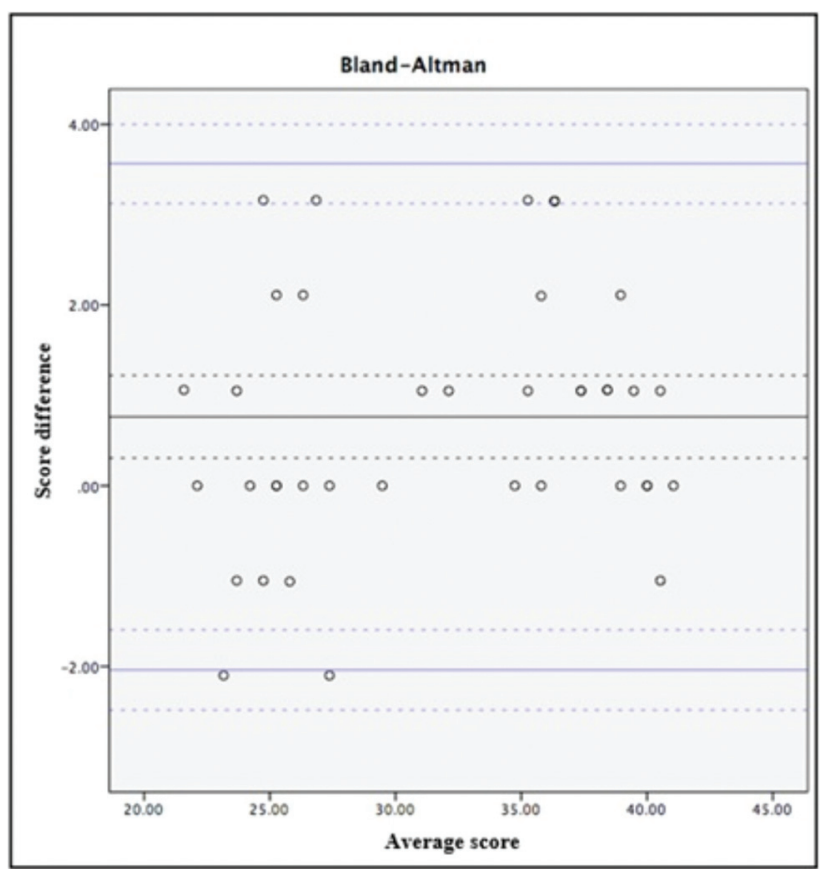

Fig. 1 Bland-Altman plot of the Bahasa Indonesia adaptation of the MDADI.

comparative. The bias of the Indonesian MDADI instrument was 0.763 , and the limit of agreement score was 2.04 (95\% confidence interval [CI]; 2.48-1.6) up to 3.57 (95\% CI; $3.12-$ 4.01). The Bland-Altman plot is presented in - Fig. 1.

\section{Discussion}

The current study involved 40 subjects, consisting of 31 men and 9 women, following a study by Carlson et al., in an MDADI adaptation to Swedish with the same characteristics, namely, 132 male and 98 female subjects. ${ }^{9}$ Research on the original MDADI version, the English version, also shows the same distribution, which is 76 male and 24 female subjects. ${ }^{6}$ The same distribution was also found in the study by Kwon et al. on the Korean language adaptation of the MDADI, namely 30 male and 3 female subjects. ${ }^{10}$ Guedes et al. adapted the MDADI study to Portuguese for a 2011 study in Brazil, with a distribution of 60 male and 12 female subjects. ${ }^{11}$

In the present study, the age distribution of subjects averaged 52.7 years (range: 19-81 years). The age group between 18 to 45 years was most common at 16 years (40\%), which is slightly different from similar studies conducted by 
Guedes et al. in 2011, in which the average age was $63+9,537 .{ }^{11}$ In the study by Carlson et al., using the Swedish adaptation of the MDADI, the average age of the subjects was 63 years, and the 55 to 64 year age group was the most common. $^{9}$

There were 38 subjects in the group with eating aids (95\%) compared with the group without eating aids, namely 2 people (5\%), in contrast to a similar study conducted by Kwon et al. in 2013 using the Korean version of the MDADI. ${ }^{10}$ In the current study, 21 subjects ate modified food, and only 6 subjects used interval aid. Carlsson et al., who tested the Swedish version of the validity and reliability of the MDADI showed 45 subjects (39.2\%) used food interval assistance. ${ }^{9}$ This difference in characteristics may be because most patients who come to the otorhinolaryngology clinic already had a hard time swallowing, so they use a food hose to help with food intake.

The dysphagia score system is divided into several scores, namely: a score of 0 indicates no dysphagia, the patient can eat normal food; score 1 indicates the patient was able to swallow some solid food; score 2 indicates the patient was able to swallow only half solid food; score 3 indicates the patient could swallow fluid only; score 4 indicates the patient could not swallow anything/total dysphagia. ${ }^{12}$ In this study, the subjects were patients with dysphagia with scores of 2 and 3, so they needed help to meet their nutritional needs.

The most common group of head and neck cancers was the nasopharyngeal cancer group, with 19 people (47.5\%). The original version of the MDADI study, in English, by Chen et al. in 2001 had a different demographic distribution, namely, the largest cancer group was the laryngeal cancer group, with 64 subjects. $^{6}$ The demographic distribution of this study is also different when compared with the study conducted by Carlson et al. with the Swedish version of MDADI, which found that the most common distribution of subjects was oropharyngeal cancer. ${ }^{9}$ The differences in demographic distribution can be explained by the fact that most of the patients with cancer who presented at the otorhinolaryngology clinic of the Dr. Sardjito Hospital had nasopharyngeal cancer. From the medical records of the Dr. Sardjito Hospital, Yogyakarta, from 1 January 2018 to 31 December 2018, we found that there were 10 patients with parotid carcinoma, with 33 visits; 1 patient with salivary gland malignanc,y with 4 visits; 21 patients with oropharyngeal carcinoma, with 58 visits; 370 patients with nasopharyngeal carcinoma, with 1,336 visits; 105 patients with sinonasal carcinoma, with 354 visits; and 73 patients with laryngeal carcinoma, with 352 visits.

Difficulty swallowing (dysphagia) affects 60 to $75 \%$ of patients treated for head and neck cancer, including those with nasopharyngeal carcinoma. Dysphagia can occur both because of the presence of the tumor and as a consequence of its treatment. Swallowing is influenced by the development of neuromuscular fibrosis after chemotherapy and radiotherapy, which causes an uncoordinated and inefficient ingestion phase that can produce long-term and continuous aspiration into the airway. Dysphagia is a major medical problem because patients may be at risk of malnutrition, dehydration, or complications due to aspiration. Dysphagia also causes a detrimental impact on the patients' quality of life. ${ }^{13}$

Dysphagia associated with oropharyngeal carcinoma often occurs posttreatment rather than pretreatment, especially when compared with the larynx and hypopharynx. Some literature shows that among patients with oral cancer and stage III to IV oropharynx, $6 \%$ suffer pretreatment dysphagia, compared with $68 \%$ in posttreatment dysphagia. ${ }^{14}$

Laryngeal cancer can occur in the supraglottis, glottis, or sub-glottis. The glottis is the most common area and is more often associated with vocal changes than dysphagia. When dysphagia occurs, there will be manifestations, such as an increase in aspiration due to vocal cord or epiglottic dysfunction during the pharyngeal swallowing process. ${ }^{14}$

Thyroid disease is a common cause of dysphagia. In one study, among patients who underwent thyroidectomy, dysphagia was the most common preoperative complaint. Thyroid disease causes pressure on the swallowing organs, which can cause symptoms from a globus sensation to dyspnea. The severity of symptoms due to suppression of the thyroid gland is directly related to the size of the gland. ${ }^{14}$

In this study, the most commonly found tumor stage group was T3 toT4, with as many as 36 people (90\%), compared with 4 people (10\%) in the T1 to T2 tumor stage group. This is in contrast to a similar study conducted by Carlson et al., using the Swedish version of the MDADI, which reported a distribution of 47 (55\%) subjects in the T0 to T2 tumor stage group, compared with 38 subjects (45\%) in the T3 to T4 tumor stage group. ${ }^{9}$ A similar study using the Brazilian version of the MDADI by Guedes et al. showed a different distribution, with as many as 40 subjects (55.6\%) in the group of subjects with a T1 to T2 clinical stage, and as many as 32 subjects (44.4\%) in the group of subjects with a T3 to T4 clinical stage. ${ }^{11}$

The most common therapeutic group was the chemotherapy and radiotherapy group, with as many as 27 (67.5\%) subjects. Studies on postchemoradiation dysphagia for head and neck cancer have found various kinds of motility disorders, including long oral transit times, decreased strength/control of the tongue, decreased contact of the tongue base to the pharyngeal wall, pharyngeal constrictor dysfunction, decreased laryngeal elevation, reduced hyoid movement, and dysmotility epiglottis. The possibility of swallowing disorders after chemoradiation appears to be related to the thickening of the pharyngeal, laryngeal, supraglottic, and glottic constrictor muscles caused by radiation. A clear relationship has been found between the distribution of doses in the salivary glands and subjective xerostomia. Chemotherapy serves as a sensitizer for radiotherapy and enhances the effects of radiotherapy on tumors and surrounding tissue. The cytotoxic effects of chemotherapy on the oral, pharyngeal, and laryngeal mucosa also cause mucositis, infection, xerostomia, and neutropenia associated with chronic dysphagia not only in head and neck cancer patients but also in patients with breast, colon, or lung cancer. ${ }^{15}$ 
The present study showed that the sub-scale interpretation of the r-value and total scale scored above 0.25 , which indicates weak-to-very strong correlation. This result means that the Indonesian MDADI questionnaire is valid for utilization. The questionnaire item with the lowest r-value was item F2, with an r-value of 0.314; however, it is still valid ( $p$-value $=0.048$ ), despite showing a weak association between the two variables. The questionnaire items with the highest r-value were P2 and E7, with an r-value of $0.939(p$-value $=0.001)$, which show a strong association between two variables.

The original version of the MDADI has been translated to various languages and cultures, such as Italian, in 2008; Swedish, in 2011; Dutch, in 2011; Korean, in 2013; Portuguese, in 2013; and Spanish, in 2019. The Italian adaptation of the MDADI was conducted by Carlson et al.; the Dutch adaptation by Speyer et al.; Korean adaptation by Kwon et al.; the Brazilian adaptation by Guedes et al.; and the Spanish adaptation by Montes-Jovellar et al. All of them showed that the items in the questionnaire are valid for use. ${ }^{9-11,16,17}$

In the present study, we performed an ICC evaluation based on a 2-way mixed effect model, mean rating $(k=2)$ and absolute consistency. The Indonesian MDADI questionnaire showed excellent reliability for the overall score result $(0.985$, 95\% CI; 0.962-0.993). The individual score domain and physical score also showed excellent reliability, while the global and functional domain showed a good-to-excellent result.

The Pearson coefficient correlation is one of the methods for evaluating the correlation between 2 tests denoted by the letter $\mathrm{r}(-1 \leq \mathrm{r} \leq 1)$, in which $\mathrm{r} \geq 0.7$ is considered as a tolerable score for questionnaire reliability. According to the study result, the r-value of each item, the domain and total score were $\geq 0.7(p<0.05)$, which indicated that the MDADI instrument is reliable.

In the Plot and Bland-Altman analyses, the MDADI instrument showed a bias score of 0.763 with a limit of agreement score of -2.04 (95\% Cl; -2.48-1.60) up to 3.57 (95\% Cl; 3.12-4.01). According to this evaluation, it can be concluded that with $95 \%$ confidence, the the MDADI measurement results will not be lower than 2.5 points if repeated population testing is performed.

\section{Conclusion}

In conclusion, we have performed the adaptation, cultural translation, and validation of the MDADI questionnaire for the Indonesian language. The Indonesian MDADI questionnaire is valid and reliable as an instrument to evaluate the quality of life of head and neck cancer patients with dysphagia. Generally, it is important to determine the characteristics and objectify concepts, such as validity and reliability, which need to be stressed when developing a questionnaire. This provides another tool to evaluate the impact of dysphagia on the quality of life of head and neck cancer patients.

\section{Author contribution}

(1) substantial contributions to conception and design, acquisition of data, or analysis and interpretation of data: Rizka Fakhriani
(2) drafting the article or revising it critically for important intellectual content: Rizka Fakhriani: Agus Surono

(3) final approval of the version to be published: Agus Surono; Bambang Udji Djoko Rianto

\section{Funding}

The present study was not supported or received any funding by any organization.

\section{Conflict of Interests}

The authors declare that there is no conflict of interests regarding the publication of this paper.

\section{Acknowledgment}

The authors would like to acknowledge Amy Y. Chen, MD, and his team for their kind permission to translate and validate the original version of the MD Anderson dysphagia inventory questionnaire into Indonesian. We would like to thank all of the staff of the Departement of otorhinolaryngology head and neck surgery, Faculty of Medicine, Public Health and Nursing, Universitas Gadjah Mada, for the support. Some results in the manuscript are from Rizka Fakhriani's thesis.

\section{References}

1 Malagelada JR, Bazzoli F, Boeckxstaens G, et al. World gastroenterology organisation global guidelines: dysphagia-global guidelines and cascades update September 2014. J Clin Gastroenterol 2015;49(05):370-378

2 Francis DO, Weymuller EA Jr, Parvathaneni U, Merati AL, Yueh B. Dysphagia, stricture, and pneumonia in head and neck cancer patients: does treatment modality matter? Ann Otol Rhinol Laryngol 2010;119(06):391-397

3 Nguyen NP, Frank C, Moltz CC, et al. Impact of dysphagia on quality of life after treatment of head-and-neck cancer. Int J Radiat Oncol Biol Phys 2005;61(03):772-778

4 Denaro N, Merlano MC, Russi EG. Dysphagia in head and neck cancer patients: Pretreatment evaluation, predictive factors, and assessment during radio-chemotherapy, recommendations. Clin Exp Otorhinolaryngol 2013;6(03):117-126

5 McHorney CA, Bricker DE, Kramer AE, et al. The SWAL-QOL outcomes tool for oropharyngeal dysphagia in adults: I. Conceptual foundation and item development. Dysphagia 2000;15(03): 115-121

6 Chen AY, Frankowski R, Bishop-Leone J, et al. The development and validation of a dysphagia-specific quality-of-life questionnaire for patients with head and neck cancer: the M. D. Anderson dysphagia inventory. Arch Otolaryngol Head Neck Surg 2001;127 (07):870-876

7 Woisard V, Andrieux MP, Puech M. [Validation of a self-assessment questionnaire for swallowing disorders (Deglutition Handicap Index)]. Rev Laryngol Otol Rhinol (Bord) 2006;127(05): 315-325

8 World Health Organization. Process of translation and adaptation of instruments. 2017 [cited 2020 May 20]. Available from: https:// www.mhinnovation.net/sites/default/files/files/WHO\%20 Guidelines\%20on\%20Translation\%20and\%20Adaptation\%20of\% 20Instruments.docx

9 Carlsson S, Rydén A, Rudberg I, Bove M, Bergquist H, Finizia C. Validation of the Swedish M. D. Anderson Dysphagia Inventory 
(MDADI) in patients with head and neck cancer and neurologic swallowing disturbances. Dysphagia 2012;27(03):361-369

10 Kwon CH, Kim YH, Park JH, Oh BM, Han TR. Validity and reliability of the korean version of the MD anderson Dysphagia inventory for head and neck cancer patients. Ann Rehabil Med 2013;37(04): 479-487

11 Guedes RL, Angelis EC, Chen AY, Kowalski LP, Vartanian JG. Validation and application of the M.D. Anderson Dysphagia Inventory in patients treated for head and neck cancer in Brazil. Dysphagia 2013;28(01):24-32

12 Ogilvie AL, Dronfield MW, Ferguson R, Atkinson M. Palliative intubation of oesophagogastric neoplasms at fibreoptic endoscopy. Gut 1982;23(12):1060-1067

13 Wilson JA, Carding PN, Patterson JM. Dysphagia after nonsurgical head and neck cancer treatment: patients' perspectives. Otolaryngol Head Neck Surg 2011;145(05):767-771
14 Moayer R, Sinha U. Dysphagia in head and neck cancer: A review. Open J Stomatol 2013;3(09):486-491. Doi: 10.4236/ojst.2013.39079

15 Rinkel RN, Verdonck-de Leeuw IM, Doornaert P, et al. Prevalence of swallowing and speech problems in daily life after chemoradiation for head and neck cancer based on cut-off scores of the patient-reported outcome measures SWAL-QOL and SHI. Eur Arch Otorhinolaryngol 2016;273(07):1849-1855

16 Speyer R, Heijnen BJ, Baijens LW, et al. Quality of life in oncological patients with oropharyngeal dysphagia: validity and reliability of the Dutch version of the MD Anderson Dysphagia Inventory and the Deglutition Handicap Index. Dysphagia 2011;26(04): 407-414

17 Montes-Jovellar L, Carrillo A, Muriel A, Barbera R, Sanchez F, Cobeta I. Translation and validation of the MD Anderson Dysphagia Inventory (MDADI) for Spanish-speaking patients. Head Neck 2019;41(01):122-129 